\title{
Preferência alimentar de Lutzomyia longipalpis (Lutz \& Neiva, 1912) em área de transmissão de leishmaniose visceral em Mato Grosso
}

\author{
Feeding preference of Lutzomyia longipalpis \\ (Lutz \& Neiva, 1912) in transmission area of \\ visceral leishmaniasis in Mato Grosso
}

\author{
Nanci Akemi Missawa ${ }^{1,2}$, Elias Seixas Lorosa ${ }^{3}$ e Edelberto Santos Dias ${ }^{2}$
}

\begin{abstract}
RESUMO
0 estudo dos hábitos alimentares e do conteúdo intestinal dos flebotomíneos permite a identificação dos hospedeiros, indicando os potenciais reservatórios das leishmanias. Este trabalho objetivou determinar a preferência alimentar de Lutzomyia longipalpis e sua relação com a transmissão da leishmaniose visceral. As capturas mensais foram realizadas em área de transmissão de leishmaniose visceral, município de Várzea Grande, Estado de Mato Grosso, no período de janeiro de 2004 a junho de 2006, utilizando-se armadilhas de luz CDC. Foram capturadas 2.376 fêmeas de Lutzomyia longipalpis, das quais 104 (4,4\%) estavam ingurgitadas, sendo 32 (30,8\%) capturadas no intradomicílio e 72 (69,2\%) no peridomicílio. Após reação de precipitina, observou-se que as fêmeas de Lutzomyia longipalpis alimentaram-se preferencialmente em aves (30,8\%) e roedores (21,2\%), mas também foram encontradas fêmeas alimentadas de sangue de humanos, gambás, bois, cavalos e cães, demonstrando o caráter oportunista da espécie.
\end{abstract}

Palavras-chaves: Lutzomyia longipalpis. Leishmaniose visceral. Preferência alimentar. Teste de precipitina.

\begin{abstract}
Studies on the feeding habits and intestinal content of sandflies make it possible to identify hosts, thereby indicating potential reservoirs for Leishmania. The present study had the aim of determining the feeding preferences of Lutzomyia longipalpis and its relationship with the transmission of visceral leishmaniasis. Specimens were caught every month in a transmission area of visceral leishmaniasis, in the municipality of Várzea Grande, State of Mato Grosso, from January 2004 to June 2006, using CDC light traps. 2,376 females of Lutzomyia longipalpis were caught, of which 104 (4.4\%) were engorged. Among these, 32 (30.8\%) were caught inside homes and $72(69.2 \%)$ in areas surrounding homes. From the precipitin reaction, it was observed that Lutzomyia longipalpis females fed preferentially on birds (30.8\%) and rodents $(21.2 \%)$, but they were also found to have fed on blood from humans, opossums, oxen, horses and dogs, thus demonstrating the opportunist nature of this species.
\end{abstract}

Key-words: Lutzomyia longipalpis. Visceral leishmaniasis. Host preference. Precipitin test.

A leishmaniose visceral (LV) possui distribuição mundial, sendo encontrada nas Américas, África, sul da Europa, Ásia e Oriente Médio ${ }^{13}$. Nas Américas, a LV ocorre desde o México até a Argentina, sendo que o Brasil contribui com 90\% dos casos do continente ${ }^{122427}$. Nesse continente, as leishmanioses têm algumas características epidemiológicas comuns, ocorrendo em pessoas residentes em áreas rurais ou que tiveram contato com habitats silvestres. As mudanças no comportamento humano, as alterações do ambiente, ou ambas, podem ter um maior impacto na prevalência e no padrão de transmissão da $\mathrm{LV}^{11}$.
No Brasil, a doença atinge as populações rurais de todos os estados, inclusive Goiás, Tocantins e Mato Grosso do Sul ${ }^{16}$, sendo que o primeiro caso humano foi registrado em 1913 em Mato Grosso $^{3}$. Apesar da conhecida subestimação de casos, o Brasil registrou um aumento na incidência da doença nos últimos 20 anos ${ }^{7}$. A leishmaniose visceral constitui um problema crescente de saúde pública no país e encontra-se em franca expansão geográfica. Levantamento feito no período de 1994 a 2002 mostrou que a doença está distribuída em 19 estados e em 1.551 municípios. Atualmente, têm sido registrados aproximadamente

\footnotetext{
1. Laboratório de Entomologia, Secretaria Estadual de Saúde de Mato Grosso, Cuiabá, MT. 2. Laboratório de Leishmanioses, Centro de Pesquisas René Rachou, Fundação Oswaldo Cruz, Belo Horizonte, MG. 3. Departamento de Entomologia, Instituto Oswaldo Cruz, Fundação Oswaldo Cruz, Rio de Janeiro, RJ.

Órgãos Financiadores: SES/MT e FIOCRUZ

Endereço para correspondência: Dr. Edelberto Santos Dias. Av. Augusto de Lima 1715, 30190-002 Belo Horizonte, MG.

Tel: 5531 3349-7700; Fax: 5531 3295-3115

e-mail: edel@cpqrr.fiocruz.br

Recebido para publicação em 24/04/2007

Aceito em 08/07/2008
} 
3.000 casos/ano no país, com letalidade média de $8 \%$ a $10 \%{ }^{10}$.

As alterações ambientais ocasionadas pelo homem e, como consequiência, a dispersão de animais silvestres que serviam como fonte de alimentação aos insetos, causam a adaptação de muitas espécies a diferentes ambientes ${ }^{9}$. A proximidade do homem a zonas de floresta e a criação de animais domésticos atraem um grande número de espécies de flebotomíneos ao peridomicílio. Uma vez atraídos, eles se estabelecem nessas áreas e representam um perigo constante como vetores de Leishmania, podendo manter o ciclo de transmissão entre animais domésticos e humanos ${ }^{51726}$.

A infecção do vetor Lutzomyia longipalpis ocorre pela ingestão, durante o repasto sanguíneo, das formas amastigostas de Leishmania (Leishmania) infantum chagasi presentes na derme do hospedeiro infectado, as quais passarão a evoluir no trato digestivo anterior do inseto para formas promastigotas ${ }^{21}$. Ao exercer o repasto sanguíneo sobre um hospedeiro não infectado, o flebotomíneo inocula as formas promastigotas infectantes presentes em seu trato digestivo anterior ${ }^{23}$, diferenciando-se em amastigotas que se disseminam pelos tecidos dos vertebrados ${ }^{30}$.

Esses insetos alimentam-se de uma grande variedade de hospedeiros vertebrados, entre eles o homem ${ }^{18}$. 0 estudo do comportamento alimentar e do conteúdo intestinal dos flebotomíneos permite a identificação dos hospedeiros, indicando os potenciais reservatórios das leishmanias.

0 presente estudo teve como objetivo determinar a preferência alimentar de Lutzomyia longipalpis e sua relação com a transmissão da LV no município de Várzea Grande, Estado de Mato Grosso.

\section{MATERIAL E MÉTODOS}

0 município de Várzea Grande (Figura 1) é a cidade industrial do Estado de Mato Grosso com população de 214.842 habitantes, extensão territorial de $949,53 \mathrm{~km}^{2}$ e altitude de $185 \mathrm{~m}$, localiza-se entre as coordenadas 1532'30" latitude sul e 56 $6^{\circ} 17^{\prime} 18^{\prime \prime}$ longitude oeste. Geograficamente, localiza-se na mesorregião centro-sul do Estado, microrregião Cuiabá e possui clima tropical quente e subúmido e precipitações de $1.750 \mathrm{~mm}$, com maior intensidade nos meses de janeiro, fevereiro e março. A temperatura média anual é de $24^{\circ} \mathrm{C}$, sendo a máxima de $42^{\circ} \mathrm{C}$, e a mínima de $0^{\circ} \mathrm{C}$. Entre as principais atividades econômicas destacam-se a indústria de transformação e comércio forte. A agricultura é de subsistência e a pecuária é pelo sistema de cria, recria e corte ${ }^{8}$.

As coletas foram realizadas em área de transmissão de LV, localizada no município de Várzea Grande, MT, no período entre janeiro de 2004 e junho de 2006, com armadilhas de luz $\mathrm{CDC}^{25}$. As armadilhas foram instaladas, mensalmente, durante quatro noites consecutivas, em 10 residências, sendo duas por residência, uma no peridomicílio (quintal e/ou abrigos de animais) e a outra no intradomicílio (quarto/dormitório).

Os insetos capturados foram sacrificados com acetato de etila e as fêmeas ingurgitadas foram congeladas a $-7^{\circ} \mathrm{C}$ para paralisação do processo digestivo. Posteriormente, foram dissecadas para triagem e confirmação da espécie Lutzomyia longipalpis. No teste de precipitina, realizado no Departamento de Entomologia do

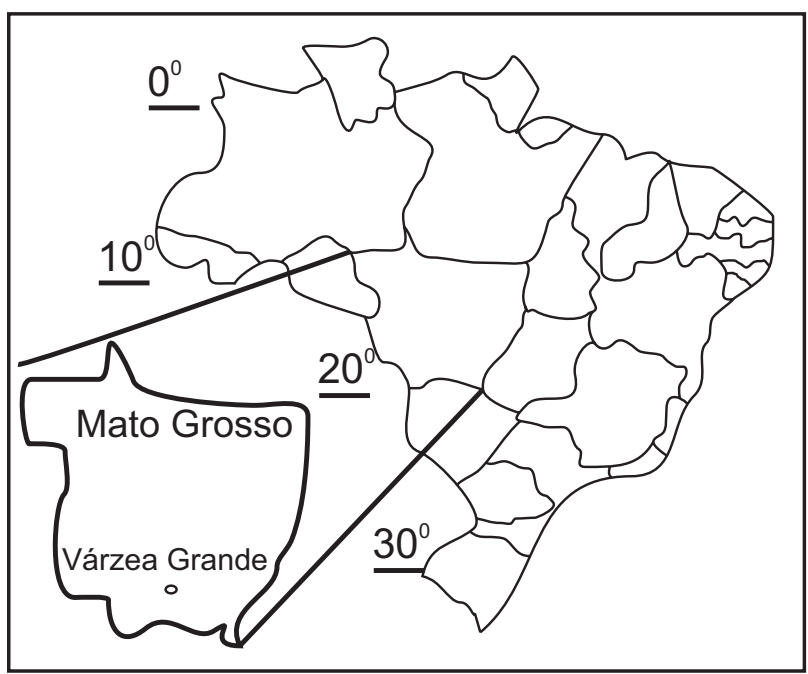

Figura 1 - Área de estudo: município de Várzea Grande, Estado de Mato Grosso, Brasil.

Instituto Oswaldo Cruz/FIOCRUZ, o conteúdo do tubo digestivo de cada fêmea foi triturado em solução salina a $0,85 \%$ e o macerado foi resfriado, durante 12 horas, à temperatura de 4 a $8^{\circ} \mathrm{C}$. Depois de centrifugado por 5 minutos a $1.500 \mathrm{rpm}$, o sobrenadante foi confrontado com anti-soros de boi, cão, cavalo, porco, roedor, ave e homem, segundo metodologia específica ${ }^{14}$.

\section{RESULTADOS}

Foram capturadas 2.376 fêmeas de Lutzomyia longipalpis no período da pesquisa, das quais 104 foram encontradas ingurgitadas, representando 4,4\%. Das 104 fêmeas de Lutzomyia longipalpis ingurgitadas, 32 (30,8\%) foram capturadas no intradomicílio e 72 (69,2\%) no peridomicílio.

A Tabela 1 apresenta a reação do conteúdo intestinal das fêmeas ao teste de precipitina, onde podemos observar a alimentação exclusiva em determinado hospedeiro ou mista em diferentes hospedeiros.

Tabela 1 - Fêmeas de Lutzomyia longipalpis submetidas ao teste de precipitina, com utilização de diferentes anti-soros, por ambiente, no município de Várzea Grande, Estado de Mato Grosso, no período entre janeiro de 2004 e junbo de 2006.

\begin{tabular}{lccc}
\hline & \multicolumn{2}{c}{ Domicílio } & \\
\cline { 2 - 3 } Anti-soros & intra & peri & Número absoluto \\
\hline Ave & 6 & 19 & 25 \\
Boi & 2 & 7 & 9 \\
Cão & 0 & 2 & 2 \\
Cavalo & 4 & 4 & 8 \\
Gambá & 6 & 6 & 12 \\
Humano & 5 & 7 & 12 \\
Roedor & 4 & 12 & 16 \\
Ave e cão & 0 & 2 & 2 \\
Ave e humano & 0 & 1 & 1 \\
Ave e roedor & 1 & 3 & 4 \\
Boi e roedor & 1 & 1 & 2 \\
Cão e humano & 0 & 1 & 1 \\
Não reagente & 3 & 7 & 10 \\
\hline Total & 32 & 72 & 104 \\
\hline
\end{tabular}


As fêmeas de Lutzomyia longipalpis alimentaram-se preferencialmente em aves $(30,8 \%)$ e roedores $(21,2 \%)$, mas também foram encontradas fêmeas alimentadas de sangue de humanos, gambás, bois, cavalos e cães (Figura 2).

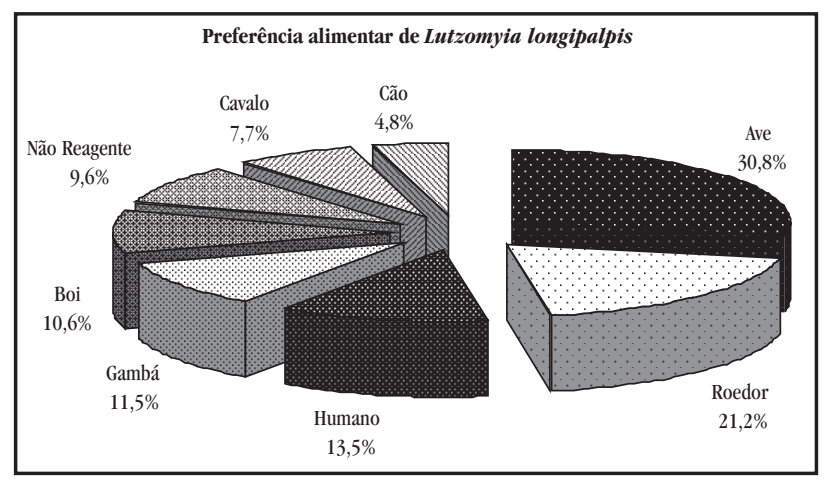

Figura 2 - Preferência alimentar de fêmeas de Lutzomyia longipalpis no município de Várzea Grande, Estado de Mato Grosso, no período entre janeiro de 2004 e junbo de 2006.

\section{DISCUSSÃo}

A busca por fontes de alimentação é uma resposta comportamental que afeta a reprodução e a densidade populacional das espécies de flebotomíneos. Assim, dependendo do seu grau de adaptação às condições ambientais modificadas pelo homem, algumas espécies podem ser mais facilmente encontradas em ambientes peridomiciliares ${ }^{4}$.

Em Várzea Grande, observou-se a preferência alimentar de Lutzomyia longipalpis por aves, sendo que essa atração também foi observada no Estado do Rio de Janeiro ${ }^{2}$ e em Porteirinha/MG ${ }^{4}$. Na Serra da Bodoquena/MS, $72 \%$ de Lutzomyia longipalpis capturados foram reagentes para aves mediante técnica imunoenzimática do Elisa ${ }^{15}$, embora a associação entre a infecção de Leishmania (Leishmania) chagasi e a presença de galinhas ou galinheiros no estado de Minas Gerais não tenha sido observada ${ }^{19}$.

0 considerável número (13,5\%) de fêmeas de Lutzomyia longipalpis alimentadas com sangue humano, seja exclusivamente ou combinado com sangue de outros animais (ave e cão), evidencia o caráter antropofilico da espécie. 0 caráter oportunista, podendo sugar ampla variedade de vertebrados, foi também relatado em Porteirinha/ $\mathrm{MG}^{4}$, onde foi observada a preferência de fêmeas de Lutzomyia longipalpis por aves e cavalos, mas também se alimentando de sangue de roedores, cães, bois e do homem. 0 caráter eclético foi observado também no município de Raposa/MA ${ }^{22}$.

Em pesquisa de preferência alimentar, com oferta de diferentes animais para o repasto sanguíneo, observou-se que 0 cavalo atraiu mais insetos que os outros animais ofertados, pois 95\% de todos os flebotomíneos capturados neste animal eram Lutzomyia longipalpis $^{28}$.

A atração de Lutzomyia longipalpis por cães foi destacada na Costa Rica ${ }^{29}$, o que não foi observado nesta pesquisa e em outros trabalhos ${ }^{20}$. Apesar da baixa porcentagem $(4,8 \%)$ de fêmeas que se alimentou em cães, a presença de gambás no peridomicílio, atuando como um elo entre os ciclos doméstico e silvestre pode aumentar 0 risco da infecção canina em 2,6 vezes 6 .

A fonte alimentar dos vetores propicia informação sobre a preferência por hospedeiros em circunstâncias naturais. A intensidade da antropofilia é um dos fatores essenciais na avaliação da capacidade vetorial, enquanto a atração por outros hospedeiros pode dar respostas sobre a associação entre vetores potenciais e reservatórios naturais ${ }^{1}$, permitindo o planejamento de estratégias de prevenção e controle da LV.

\section{AGRADECIMENTOS}

Agradecemos aos técnicos do Laboratório de Entomologia e do Escritório Regional de Saúde da Baixada Cuiabana da Secretaria de Saúde de Mato Grosso pelos trabalhos de campo e aos moradores das casas pesquisadas no município de Várzea Grande/MT pela valiosa colaboração.

\section{REFERÊNCIAS}

1. Afonso MMS, Gomes AC, Meneses CRV, Rangel EF. Studies on the feeding habits of Lutzomyia (N.) intermedia (Diptera, Psychodidae), vector of cutaneous leishmaniasis in Brazil. Cadernos de Saúde Pública 21:1816-1820, 2005.

2. Aguiar GM, Vilela ML, Lima RB. Ecology of the sandflies of Itaguaí, in area of cutaneous leishmaniasis in the state of Rio de Janeiro. Feed preferences (Diptera, Psychodidae, Phlebotominae). Memórias do Instituto Oswaldo Cruz 82: 583-584, 1987.

3. Alencar JE, Dietze R. Leishmaniose visceral (Calazar). In: Veronesi R. Doenças infecciosas e parasitárias. $8^{a}$ edição. Editora Guanabara Koogan. Rio de Janeiro, p. 706-717, 1991.

4. Barata RA, França-Silva JC, Mayrink W, Silva JC, Prata A, Lorosa ES, Fiúza JA, Gonçalves CM, de Paula KM, Dias ES. Aspectos da ecologia e do comportamento de flebotomíneos em área endêmica de leishmaniose visceral, Minas Gerais. Revista da Sociedade Brasileira de Medicina Tropical 38: 421-425, 2005.

5. Barbosa GM, Marzochi MCA, Massard CL, Lima GPS, Confort EM. Epidemiological aspects of canine american tegumentary leishmaniasis in the municipality of Paraty, State of Rio de Janeiro, Brazil. Cadernos de Saúde Pública 15:641-646, 1999.

6. Cabrera MAA, De Paula AA, Camacho LAB, Marzochi CA, Aguiar GM, Xavier SC, Silva AVM, Jansen AM. Canine Visceral Leishmaniasis in Barra de Guaratiba, Rio de Janeiro, Brazil: assessment of some risk factors. Revista do Instituto de Medicina Tropical de São Paulo 45:79-83, 2003.

7. Dantas-Torres F, Brandão-Filho SP. Visceral leishmaniasis in Brazil: revisiting paradigms of epidemiology and control. Revista do Instituto de Medicina Tropical de São Paulo 48:151-156, 2006.

8. Ferreira JCV. Mato Grosso e seus Municípios. Editora Buriti. Cuiabá/MT, 2001.

9. Gomes AC, Barata JM, Rocha e Silva EO, Galati EA. Ecologic aspects of american tegumentary leishmaniasis. 6. Anthropophilic Phlebotomus fauna of residual forests located in the northeastern region of the state of São Paulo, Brazil. Revista do Instituto de Medicina Tropical de São Paulo 31:32-39, 1989.

10. Gontijo CMF, Melo MN. Leishmaniose Visceral no Brasil: quadro atual, desafios e perspectivas. Revista Brasileira de Epidemiologia 7:338-349, 2004.

11. Grimaldi Jr G, Tesh RB. Leishmaniasis of the New World: current concepts and implications for future research. Clinical Microbiology Reviews 6:230-250, 1993.

12. Grimaldi Jr G, Tesh RB, Pratt DM. A review of geographical distribution and epidemiology of leishmaniasis in the New World. Clinical Microbiology Reviews 41:687-725, 1989. 
13. Lainson R, Shaw JJ. Evolution, classification and geographical distribution. In: Peters W, Killick-Kendrick R (eds) The leishmaniasis in biology and medicine, London. Academic Press 1: p.1-20, 1987.

14. Lorosa ES, Andrade RE. Identificação de fontes alimentares de mosquitos no município de Nova Iguaçu, RJ, Brasil, pela técnica da reação de precipitina. Entomología y Vectores 5:85-92, 1998.

15. Marassá AM, Consales CA, Galati EAB, Nunes VLB. Identificação do sangue ingerido por Lutzomyia (Lutzomyia) longipalpis (Lutz \& Neiva, 1912) e Lutzomyia (Lutzomyia) almerioi (Galati \& Nunes, 1999) pela técnica imunoenzimática do ELISA de captura, no sistema avidina-biotina. Revista da Sociedade Brasileira de Medicina Tropical 39:183-186, 2006.

16. Marzochi MCA, Marzochi KBF. Tegumentary and visceral leishmaniasis in Brazil - Emerging anthropozoonosis and possibilites for their control. Cadernos de Saúde Pública 10:359-375, 1994.

17. Ministério da Saúde. Manual de Vigilância e Controle da Leishmaniose Visceral. Fundação Nacional de Saúde, Brasília, 2003.

18. Monteiro EM, França-Silva JC, Costa RT, Costa DC, Barata RA, Paula EV, MachadoCoelho GLL, Rocha MF, Fortes-Dias CL, Dias ES. Leishmaniose visceral: estudo de flebotomíneos e infecção canina em Montes Claros, Minas Gerais. Revista da Sociedade Brasileira de Medicina Tropical 38:147-152, 2005.

19. Moreno EC, Melo MN, Genaro 0, Lambertucci JR, Serufo JC, Andrade ASR, Antunes CMF, Carneiro MC. Risk factors for Leishmania chagasi infection in an urban area of Minas Gerais state. Revista da Sociedade Brasileira de Medicina Tropical 38: 456-463, 2005.

20. Morrison AC, Ferro C, Tesh RB. Host preferences of the sandfly at an endemic focus of American Visceral Leishmaniasis in Colombia. The American Journal of Tropical Medicine and Hygiene 49: 68-75, 1993.
21. Neves DP. Parasitologia Humana. $11^{\mathrm{a}}$ edição. Editora Atheneu. São Paulo, 2004.

22. Passos-Dias FO, Lorosa EL, Rebelo JMM. Fonte alimentar sanguínea e a peridomiciliação de Lutzomyia longipalpis (Lutz \& Neiva, 1912) (Psychodidae, Phlebotominae). Cadernos de Saúde Pública 19:1373-1380, 2003.

23. Sacks DL. Metacyclogenesis in Leishmania promastigotes. Experimental Parasitology 69: 100-103, 1989.

24. Soares RPP, Turco SJ. Lutzomyia longipalpis (Diptera: Psychodidae: Phlebotominae) a review. Anais da Academia Brasileira de Ciências 75: 303-330, 2003.

25. Sudia WA, Chamberlain RW. Battery-Operated light trap: an improved model. Mosquitoes News 22:126-129, 1962.

26. Vexenat JA, Barreto AC, Cuba CC, Marsden PD. Epidemiological characteristics of american cutaneous leishmaniasis in an endemic region of the state of Bahia. III. Phlebotominae fauna. Memórias do Instituto Oswaldo Cruz 81:293-301, 1986.

27. World Health Organization. Lucha contra las leishmaniasis. Série Informe Técnico $793,1990$.

28. Ximenes MFFM, Souza MF, Castellón EG. Density of sand flies (Diptera: Psychodidae) in domestic and wild animal shelters in an area of visceral leishmaniasis in the state of Rio Grande do Norte, Brazil. Memórias do Instituto Oswaldo Cruz 94:427-432, 1999.

29. Zeledon R, Murrillo J, Gutierrez H. Observaciones sobre la ecologia de Lutzomyia longipalpis (Lutz \& Neiva, 1912) y possibilidades de existencia de leishmaniasis visceral en Costa Rica. Memórias do Instituto Oswaldo Cruz 79: 455-459, 1984

30. Zilberstein D, Shapira M. The role of $\mathrm{pH}$ and temperatura in development of Leishmania parasites. Annual Review of Microbiology 48: 449-470, 1994. 